\title{
Treatment Session Frequency and Smoking Cessation
}

\author{
Joy M. Schmitz \\ University of Texas Medical School, Houston \\ James C. Tate \\ University of Michigan
}

\begin{abstract}
In a retrospective program evaluation analysis, we compared abstinence rates in 78 smokers participating in a 2-, 3-, or 6-week clinic-based behavioralpharmacological smoking cessation program. The three treatment formats consisted of six sessions that varied in session frequency. Retention and end-oftreatment smoking cessation rates were best in the medium-frequency (3-week) and high-frequency (2-week) treatment session formats. One-year follow-up abstinence rates were highest in the medium-frequency format, and lowest in the highfrequency format. A significant patient-treatment interaction effect was found for pretreatment smoking rate. Individuals with lower pretreatment smoking rates benefited most with respect to end-of-treatment cessation rates from the medium-and high-frequency treatment session format. However, this effect was not significant at the 1-year follow-up. The need for prospective research and patient-to-treatment matching implications in smoking cessation programs are discussed.
\end{abstract}

A large body of literature exists on various methods used to help smokers quit successfully (Lichtenstein \& Glasgow, 1992). In recent years, the focus of smoking intervention research has shifted from the development of new, innovative techniques to the refinement of various treatment components. There is growing interest in the possibility of improving treatment outcome by matching smokers to treatments designed to meet their specific needs (e.g., Stevens \& $\mathrm{Hol}$ lis, 1989).

A popular clinic-based smoking cessation treatment is one that employs behavioral self-regulation and relapse prevention training with adjunctive pharmacological therapy (Carmody, 1992). Studies that have examined ways to optimize this treatment approach have focused on a variety of "therapeutic" components, such as type of skills trained (Emmons, Emont, Collins, \& Weidner, 1988) and schedule of nicotine gum administration (Goldstein, Niaura, Follick, \& Abrams, 1989). Another set of potential matching variables are those associ-

This project was supported in part by a Biomedical Research Support Grant to Joy Schmitz. Special thanks are due to Burnice Marshall and Tao Le for their assistance in coordinating and collecting the data for this research.

Correspondence and requests for reprints should be sent to Joy M. Schmitz, Substance Abuse Research Center, UT Mental Sciences Institute, 1300 Moursund Ave., Houston, TX 77030. 
ated with how the treatment process is organized and presented to clients. Decisions regarding treatment delivery factors are often based on pragmatic and economic considerations, rather than empirical evidence. One notable exception is the work of Lando (1987), who evaluated the relative effectiveness of lay versus professional facilitators in conducting smoking cessation programs.

This article presents findings that emerged from a retrospective smoking cessation program evaluation analysis. The clinic-based behavioral-pharmacological program for smoking cessation included six treatment sessions that, due to practical considerations, were delivered in 6 weeks ( 1 session per week), 3 weeks ( 2 sessions per week), or 2 weeks ( 3 sessions per week). Although treatment content remained the same, the frequency with which these sessions were delivered emerged as a significant predictor of dropout, initial quit rate, and 1-year sustained abstinence. In addition to treatment format, we examined other variables that might interact with format to predict treatment success.

\section{METHOD}

\section{Subjects}

Smokers were recruited through posted announcements advertising a Tobacco Independence Program (TIP). Seventy-eight persons enrolled in the program. This sample consisted of 33 men and 43 women. Table 1 contains data on pretreatment smoking history characteristics. Generally, the sample consisted of moderately heavy and dependent smokers who had been smoking more than 5 years.

\section{Measures}

A smoking history questionnaire was used to assess smoking-relevant variables and included a 1 -item motivation to quit smoking scale $(0=$ not at all to $8=$ extremely). The Fagerström Tolerance Questionnaire (FTQ; Fagerström, 1978) was administered as a measure of nicotine dependence (scale range $=1-11$ ). The Smoking Self-Efficacy Questionnaire (SSEQ; Colletti, Supnick, \& Payne, 1985) was used to measure self-efficacy regarding not smoking in various highrisk situations (score range: 0-100). The 14-item Perceived Stress Scale (PSS; Cohen, Kamarck, \& Mermelstein, 1983) measured the degree to which individuals appraised situations in their lives as stressful (score range: 0-56). PSS scores have been found to be lower for quitters than for nonquitters in studies predicting successful outcome in smoking cessation (Glasgow, Klesges, Mizes, \& Pechacek, 1985).

Alveolar carbon monoxide (CO) levels were measured with the MiniCO Model 1000 portable ecolyzer (Catalyst Research Corporation, Owings Mills, MD). A $\mathrm{CO} \leq 10 \mathrm{ppm}$ was used to indicate abstinence. Clients self-recorded daily smoking rate using small tally cards. These two measurements were collected at the start of each treatment session. 


\section{Procedure}

All potential subjects participated in an initial telephone interview designed to explain the treatment program and evaluate eligibility to participate. To enroll in the program, individuals had to be smoking at least 10 cigarettes per day, lacking severe psychiatric or medical problems, and able to attend six treatment sessions. As this was not a prospective study, patients were not assigned randomly to treatment groups. The procedure to assign cohorts to the treatment formats (6-, 3-, 2-week) was nonrandom and took into account staffing and space availability. Nevertheless, subjects did not self-select treatment format, and no patient characteristic determined format assignment.

\section{Treatment}

The TIP smoking treatment consisted of six 1-hour sessions delivered in group format (up to eight participants per group) by trained doctoral-level clinical psychology interns. Group leaders were supervised on a weekly basis by the first author. A detailed, session-by-session treatment manual was used by the group leaders. The smoking intervention employed in this study is representative of those currently offered in most formal cessation clinics. The structured behavioral-pharmacological treatment approach highlights four components: gradual nicotine fading, coping skills training, relapse prevention strategies, and nicotine replacement therapy (Nicotine Polacrilex) as a pharmacological treatment adjunct. Participants were instructed to select a target quit date between Sessions 4 and 5 .

The treatment program was delivered in three formats that differed with respect to the frequency of sessions. In the low-frequency (LF) format, subjects participated in six weekly sessions. A total of 22 subjects were treated in this format, involving five cohorts over a 30 -week period. In the medium-frequency (MF) format, the six treatment sessions were conducted over a 3-week period. The MF group consisted of a total of 29 subjects, in six cohorts over a 32-week period. Finally, the high-frequency (HF) format involved six sessions conducted over a 2-week period. A total of 27 subjects were treated in the HF format (six cohorts over a 24-week period). Follow-up data collection sessions were held approximately 12 months ( $r$ : 12-14 months) after the end of treatment. Subjects who completed treatment were asked to return for a follow-up visit. Daily smoking rate and measurement of smoking status were verified by $\mathrm{CO}$ levels.

\section{RESULTS}

\section{Comparability of Conditions}

Univariate analyses of variance (ANOVAs) for continuous measures and chisquare analyses for frequency variables revealed that there were no significant differences between groups on demographic or pretreatment smoking history 
Table 1. Subject Pretreatment Characteristics by Treatment Format

\begin{tabular}{|c|c|c|c|}
\hline Characteristic & $\begin{array}{c}\text { LF } \\
(N=22)\end{array}$ & $\begin{array}{c}\text { MF } \\
(N=29) \\
\end{array}$ & $\begin{array}{c}\text { HF } \\
(N=27)\end{array}$ \\
\hline \multicolumn{4}{|l|}{ Gender } \\
\hline Male & 11 & 11 & 11 \\
\hline Female & 11 & 18 & 16 \\
\hline \multicolumn{4}{|l|}{ Ethnicity } \\
\hline Caucasian & 19 & 20 & 20 \\
\hline Other & 3 & 9 & 7 \\
\hline \multicolumn{4}{|l|}{ Marital Status } \\
\hline Married & 8 & 12 & 8 \\
\hline Divorced/Separated & 10 & 8 & 10 \\
\hline Single & 4 & 9 & 9 \\
\hline \multicolumn{4}{|l|}{ Employment } \\
\hline Employed & 15 & 21 & 23 \\
\hline Unemployed & 7 & 8 & 4 \\
\hline \multicolumn{4}{|l|}{ Education (years) } \\
\hline$M$ & 14.36 & 15.06 & 15.55 \\
\hline$S D$ & 2.40 & 3.18 & 2.50 \\
\hline \multicolumn{4}{|l|}{ Age } \\
\hline$M$ & 39.36 & 42.13 & 40.87 \\
\hline$S D$ & 7.22 & 10.96 & 9.75 \\
\hline \multicolumn{4}{|l|}{ Cigarettes/day } \\
\hline$M$ & 30.50 & 23.67 & 24.92 \\
\hline$S D$ & 14.70 & 11.72 & 12.97 \\
\hline \multicolumn{4}{|l|}{ Years Smoked } \\
\hline$M$ & 16.40 & 19.58 & 16.36 \\
\hline$S D$ & 9.75 & 11.87 & 8.57 \\
\hline \multicolumn{4}{|l|}{ Longest Abstinence (days) } \\
\hline$M^{\circ}$ & 103.68 & 235.03 & 122.18 \\
\hline$S D$ & 159.69 & 337.21 & 262.67 \\
\hline \multicolumn{4}{|c|}{ Nicotine Dependence (TQ score) } \\
\hline$M$ & 7.76 & 7.75 & 6.94 \\
\hline$S D$ & 1.89 & 1.74 & 1.95 \\
\hline \multicolumn{4}{|l|}{ Motivation to Quit } \\
\hline$M$ & 6.36 & 6.93 & 6.20 \\
\hline$S D$ & 1.55 & 1.27 & 1.89 \\
\hline \multicolumn{4}{|l|}{ Self-Efficacy (SSEQ score) } \\
\hline$M$ & 47.36 & 44.74 & 52.31 \\
\hline$S D$ & 18.06 & 19.01 & 15.37 \\
\hline \multicolumn{4}{|l|}{ Stress (PSS score) } \\
\hline$M$ & 26.72 & 24.96 & 24.45 \\
\hline$S D$ & 6.43 & 7.70 & 6.75 \\
\hline
\end{tabular}

Note. $\mathrm{LF}=$ low frequency; $\mathrm{MF}=$ medium frequency; $\mathrm{HF}=$ high frequency; $\mathrm{TQ}=$ Tolerance Questionnaire; SSEQ = Smoking Self-Efficacy Questionnaire; PSS $=$ Perceived Stress Scale.

measures. There was a nonsignificant trend for subjects in the MF group to report longer past abstinence periods; however, this apparent difference was caused by data from two subjects with extreme values (e.g., longest abstinence $\geq$ 5 years). Subsequent analyses, performed with and without removal of these outliers, yielded similar results. Descriptive information on these variables by treatment format is shown in Table 1 . 
Table 2. Treatment Retention, Quit Rates, and 1-Year Outcomes

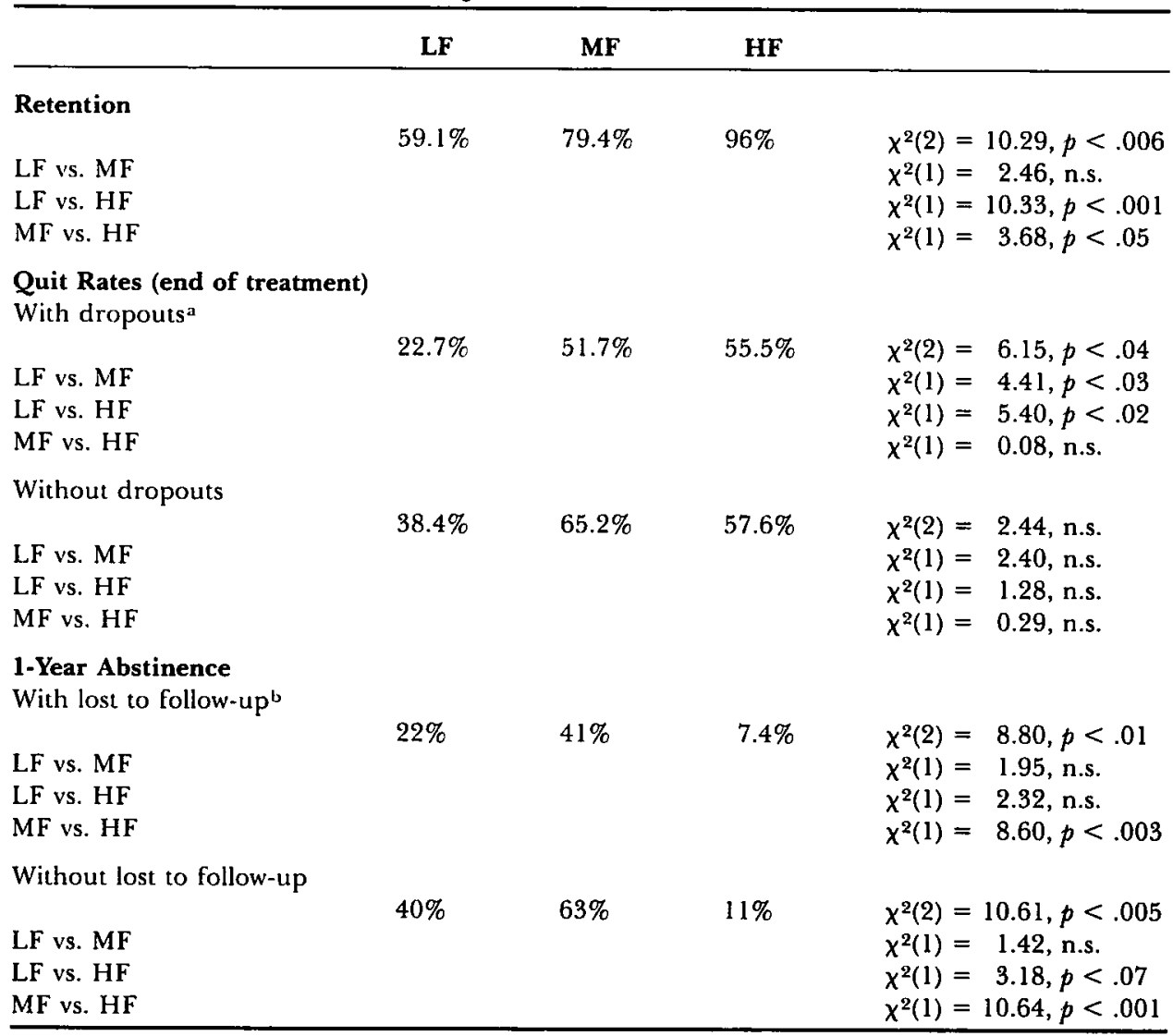

Note. $\mathrm{LF}=$ low frequency; $\mathrm{MF}=$ medium frequency; $\mathrm{HF}=$ high frequency.

a With dropouts included and classified as treatment failures.

bWith subjects lost to follow-up included and classified as treatment failures.

\section{Treatment Retention, Smoking Reduction, and Quitting}

Subjects were considered to have completed treatment if they attended $50 \%$ of the treatment-that is, at least three of the six sessions. Significantly more subjects receiving the LF and MF formats dropped out of treatment compared to those receiving the HF format (see Table 2). Reanalyses of group differences on demographic and pretreatment smoking variables, with dropouts excluded, indicated that the three treatment groups remained comparable.

To test for between-group differences in smoking rate reduction during treatment, daily smoking rates were calculated as a percentage of the pretreatment rate, and a two-factor (format and session) analysis of variance with repeated measures on one factor (session) was performed. Subjects who dropped out of treatment were excluded from this analysis. Repeated ANOVA results showed a significant decrease in smoking rate over sessions, $F(4,96)=75.5, p<.0001$, a significant main effect for format, $F(2,96)=4.73, p<.01$, and a significant session by format interaction, $F(8,96)=2.04, p<.04$. Tukey post hoc compari- 
sons indicated that subjects in the MF group had significantly lower smoking rates at treatment Session 3 than LF and HF subjects. Significant differences between treatment conditions also were found at Session 5, with both MF and HF subjects reporting lower smoking rates than LF subjects.

End-of-treatment abstinence rates between the LF, MF, and HF treatment format groups were not significantly different but clearly favored the shorter formats (see Table 2). The same analysis performed with dropouts included and classified as treatment failures yielded significant results, with higher abstinence rates found in the HF and MF formats as compared to the LF format (see Table 2).

\section{One-Year Outcome}

Sustained abstinence at the 1-year follow-up assessment was defined as a selfreport of no tobacco use in the previous 12 months and $\mathrm{CO} \leq 10 \mathrm{ppm}$. Thirty subjects were lost to follow-up (38\%). The lost to follow-up rate did not differ significantly between the three formats $(\mathrm{LF}=45.4 \%, \mathrm{MF}=34.4 \%, \mathrm{HF}=37 \%$; $\chi^{2}(2)=.73$, n.s.).

The number of abstinent smokers at 1-year posttreatment was dependent upon treatment format (see Table 2). More subjects in the MF format were abstinent at follow-up than in the HF format. Abstinence rates of the MF and LF formats were not significantly different but clearly favored the MF format. The same pattern of. results was obtained when subjects lost to follow up were included as treatment failures in the analysis. Again, more subjects in the MF format were abstinent than in the HF format. Differences between the MF and LF formats were not significant.

\section{Use of Nicotine Gum}

Nicotine Polacrilex (Nicorette, Marion Merrell Dow Pharmaceuticals) was available as a temporary adjunct to group treatment. When used this way, most studies show that nicotine gum increases long-term quit rates (e.g., Hall, Tunstall, Rugg, Jones, \& Benowitz, 1985; Hjalmarson, 1984; Killen, Fortmann, Newman, \& Varady, 1990). Thirty-five subjects (56\%) used nicotine gum. Chisquare analyses indicated no significant differences in gum use between the LF, $M F$, and HF treatment formats, $\chi^{2}(2)=.73$, n.s., or between abstinent and relapsed smokers at 1 -year follow-up, $\chi^{2}(1)=1.01$, n.s.

\section{Patient-Treatment Interactions}

Hierarchical logistic regression analyses (Kelsey, Thompson, \& Evans, 1986) were used to test whether any of the patient characteristics (pretreatment smoking rate, nicotine dependence, motivation, self-efficacy, stress) interacted with treatment format to predict dichotomously scored outcomes (smoking status at end-of-treatment and 1-year follow-up).

In the first regression analysis, the predictors entered were pretreatment smoking rate, treatment format, and the treatment format by pretreatment smoking rate. The interaction of Pretreatment Smoking Rate $\times$ Treatment For- 
mat significantly predicted end-of-treatment smoking status, improvement $\chi^{2}(2)$ $=10.9, p<.01$; goodness-of-fit $\chi^{2}(72)=75.8, p=.35$. For those patients assigned to the MF and HF groups, the probability of quitting decreased as pretreatment smoking rate increased. For those patients who received the LF format, end-of-treatment outcome was unaffected by pretreatment smoking rate. The interaction of treatment format with nicotine dependence (TQ score) did not predict end-of-treatment cessation status. There were no significant interactions for pretreatment smoking rate or nicotine dependence when logistic regression models were used to predict 1-year outcomes. Psychological variables (motivation, self-efficacy, stress) failed to predict any of the outcome measures in an interaction with treatment type.

\section{DISCUSSION}

The study presented here evaluated a clinic-based behavioral-pharmacological smoking cessation treatment delivered in three formats of varying session frequency. Individuals receiving the LF format showed smaller smoking reductions and were more likely to drop out over the course of treatment. Retention in treatment and end-of-treatment abstinence rates clearly favored the shorter formats. One-year abstinence rates, with and without dropouts counted as failures, were highest in the MF format as compared to the LF and HF formats. All other factors being equal, the MF format appeared to augment treatment outcome.

A significant patient-treatment interaction effect was found for pretreatment smoking rate. The low-frequency treatment session format appeared to be equally effective, regardless of pretreatment smoking level. On the other hand, the medium- and high-frequency treatment session formats were most successful in inducing abstinence among those considered "lighter" smokers at pretreatment. These results have implications for matching in clinic practice. A more rapid rate reduction and quitting approach may be more appropriate for those individuals who enter treatment smoking fewer cigarettes; conversely, a slower, more gradual smoking cessation format may be detrimental to their cessation efforts. Because we did not collect sufficiently detailed data on group process variables, it is unknown how the shorter formats, especially the MF format, effected their influence. More frequent treatment sessions may have facilitated the development of greater group cohesiveness, friendship, and support, thereby explaining the greater success in initial smoking reduction and quitting for the MF and HF formats.

An alternative explanation relates to readiness for behavior change. The present sample was comprised of patients who scored fairly high on self-efficacy and perceived low stress. Moreover, the sample as a whole was highly motivated to quit smoking and could be conceptualized as being in what has been called the action stage (Prochaska, DiClemente, \& Norcross, 1992). Prepared for action, smokers highly motivated to quit may experience a "critical period" during which their chances for initial cessation are optimal, possibly owing to high motivation. For the LF format subjects, this critical period may have passed prior to their scheduled quit dates.

Why so many HF format subjects initially quit only to relapse as compared to 
subjects in the LF format (low quit and low relapse rates) and MF format (high quit and low relapse rates) is unknown. It is possible that some LF subjects dropped out of treatment because their initial motivation waned; this suggests that subjects who completed treatment were more motivated to quit and survived a lengthy 4-week weeding out process. Such individuals might be less likely to relapse. Likewise, the HF format's brevity may have enabled even the least motivated smokers to quit initially only to relapse later because of an inadequate interval of postcessation supervision. The MF format may have exerted its effects via a combination of the benefits of the LF and HF formats. That is, it could have been sufficiently short to enable less motivated subjects to quit initially and sufficiently long to provide sufficient immediate postcessation support.

Study limitations must be considered when interpreting the present findings. First and foremost, this study's design was not experimental and did not involve random assignment of subjects to treatment formats. Consequently, statements concerning causality are tentative and speculative. Nevertheless, subjects did not self-select treatment format, no subject characteristic determined format assignment, and the groups were comparable on demographic and smoking history variables. Second, we utilized carbon monoxide analysis for verifying 1-year smoking status. Although this procedure is a methodological improvement over self-report only, one disadvantage is CO's relatively short half-life, approximately $\mathbf{4}$ hours, compared to other more sensitive biochemical indices of smoking, such as cotinine assays (Benowitz, 1983). However, it is unlikely that nonabstinent subjects reporting at the l-year follow-up would abstain for the necessary length of time simply to lower their CO measurement. Third, the TIP treatment was offered in a large medical center located within a large metropolitan area. Subjects were either medical center employees or lived in the metropolitan area and were less inconvenunced by session frequency than subjects living in more rural areas who would have had to travel longer distances to attend treatment sessions. Consequently, the present results may not apply to such populations. Fourth, the sample size was limited and precluded the examination of gender effects. Finally, the high attrition rate may be problematic; however, dropout was a dependent variable expected to differ based on treatment format. We opted for a conservative definition of dropouts as "treatment failures"; yet the validity of this assumption cannot be determined.

At the very least, the present results highlight the importance of comprehensive data collection, follow-up, and program evaluation in commercial smoking cessation programs. Although our results may not generalize to all smoking cessation programs, they appear valid in our program's setting. We have adopted the medium-frequency format exclusively because we get generally better results when using it. To the extent that the results of smoking cessation research become incorporated into commercial programs (and program formats change as a result), periodic evaluation of treatment success is important to determine whether new components augment, truncate, or have neutral effects on treatment success in a given setting.

Intensive multisession group programs probably will continue to serve a minority of smokers. Identifying patient characteristics that are most related to 
favorable outcome, and then using this information to assign patients to the most appropriate treatments, should increase the overall effectiveness of smoking intervention programs. Untangling complex patient-program interactions is a major challenge facing researchers in the treatment of addictive behaviors. This study's retrospective results highlight the need for prospective investigations of the effects of treatment characteristics.

\section{REFERENCES}

Benowitz, N.L. (1983). The use of biologic fluid samples in assaying tobacco smoke consumption. In J. Grabowski \& C. Bell (Eds.) Measurement in the analysis and treatment of smoking behavior (National Institute on Drug Abuse Research Monograph 48, pp. 6-26. DHHS Publication No. ADM 83-1285). Washington, DC: Superintendent of Documents, U.S. Government Printing Office.

Carmody, T.P. (1992). Preventing relapse in the treatment of nicotine addiction: Current issues and future directions. Journal of Psychoactive Drugs, 24, 13I-158.

Cohen, S., Kamarck, T., \& Mermelstein, R. (1983). A global measure of perceived stress. Journal of Health and Social Behavior, 24, 385-396.

Colletti, G., Supnick, J.A., \& Payne, T.J. (1985). The smoking self-efficacy questionnaire (SSEQ): Preliminary scale development and validation. Behavioral Assessment, 7, 249-260.

Emmons, K.M., Emont, S.L., Collins, R.L., \& Weidner, G. (1988). Relapse prevention versus broad spectrum treatment for smoking cessation: A comparison of efficacy. Journal of Substance Abuse, 1, 79-90.

Fagerström, K.O. (1978). Measuring degree of physical dependence on tobacco smoking with reference to individualization of treatment. Addictive Behaviors, 3, 235-24l.

Glasgow, R.E., Klesges, R.C., Mizes, J.S., \& Pechacek, T.F. (1985). Quitting smoking: Strategies used and variables associated with success in a stop-smoking contest. Journal of Consulting and Clinical Psychology, 53, 905-912.

Goldstein, M.G., Niaura, R., Follick, M.J., \& Abrams, D.B. (1989). Effects of behavioral skills training and schedule of nicotine gum administration on smoking cessation. American Journal of Psychiatry, 146, 56-60.

Hall, S.M., Tunstall, C., Rugg, D., Jones, R.T., \& Benowitz, N. (1985). Nicotine gum and behavioral treatment in smoking cessation. Journal of Consulting and Clinical Psychology, 53, 256-258.

Hjalmarson, A. (1984). Effect of nicotine chewing gum in smoking cessation: A randomized, placebo-controlled, double-blind study. Journal of the American Medical Association, 252, 28352838.

Kelsey, J.L., Thompson, W.D., \& Evans, A.S. (1986). Methods in observational epidemiology. New York: Oxford University Press.

Killen, J.D., Fortmann, S.P., Newman, B., \& Varady, A. (1990). Evaluation of a treatment approach combining nicotine gum with self-guided behavioral treatments for smoking relapse prevention. Journal of Consulting and Clinical Psychology, 58, 85-92.

Lando, H.A. (1987). Lay facilitators as effective smoking cessation counselors. Addictive Behaviors, 12, 69-72.

Lichtenstein, E., \& Glasgow, R.E. (1992). Smoking cessation: What have we learned over the past decade? Journal of Consulting and Clinical Psychology, 60, 518-527.

Prochaska, J.O., DiClemente, C.C., \& Norcross, J.C. (1992). In search of how people change: Applications to addictive behaviors. American Psychologisl, 47, 1102-1114.

Stevens, V.J., \& Hollis, J.F. (1989). Preventing smoking relapse using an individually tailored skillstraining technique. Journal of Consulting and Clinical Psychology, 57, 420-424. 